\title{
A Literature Review on Cloud Computing Adoption Issues in Enterprises
}

\author{
Rania Fahim El-Gazzar \\ Department of Information Systems, University of Agder, Kristiansand, Norway \\ rania.f.el-gazzar@uia.no
}

\begin{abstract}
Cloud computing has received increasing interest from enterprises since its inception. With its innovative information technology (IT) services delivery model, cloud computing could add technical and strategic business value to enterprises. However, cloud computing poses highly concerning internal (e.g., Top management and experience) and external issues (e.g., regulations and standards). This paper presents a systematic literature review to explore the current key issues related to cloud computing adoption. This is achieved by reviewing 51 articles published about cloud computing adoption. Using the grounded theory approach, articles are classified into eight main categories: internal, external, evaluation, proof of concept, adoption decision, implementation and integration, IT governance, and confirmation. Then, the eight categories are divided into two abstract categories: cloud computing adoption factors and processes, where the former affects the latter. The results of this review indicate that enterprises face serious issues before they decide to adopt cloud computing. Based on the findings, the paper provides a future information systems (IS) research agenda to explore the previously under-investigated areas regarding cloud computing adoption factors and processes. This paper calls for further theoretical, methodological, and empirical contributions to the research area of cloud computing adoption by enterprises.
\end{abstract}

Keywords: Cloud computing, adoption, enterprise.

\section{$1 \quad$ Introduction}

Over the past decade, there has been a heightened interest in the adoption of cloud computing by enterprises. Cloud computing promises the potential to reshape the way enterprises acquire and manage their needs for computing resources efficiently and cost-effectively [1]. In line with the notion of shared services, cloud computing is considered an innovative model for IT service sourcing that generates value for the adopting enterprises [2]. Cloud computing enables enterprises to focus on their core business activities, and, thus, productivity is increased [3]. The adoption of cloud computing is growing rapidly due to the scalability, flexibility, agility, and simplicity it offers to enterprises [3-6]. A recent cross-sectional survey by [7] on the adoption rates of cloud computing by enterprises reported that $77 \%$ of large enterprises are adopting the cloud, whereas $73 \%$ of small and medium-sized enterprises (SMEs) are adopting the cloud. 
Cloud computing is "an old idea whose time has (finally) come" [8]. The term cloud is old since it was drawn in network diagrams as a metaphor representing the Internet [9]. Cloud computing is generally referred to as providing "Internet-based computing service" [10]; however, the technical meaning is richer, as cloud computing builds on already-existing computing technologies, such as grid computing and virtualization, which are forms of distributed computing technology [9]. Virtualization involves masking the physical characteristics of computing resources to hide the complexity when systems, applications, or end users interact with them [9]. Grid computing is "a model of distributed computing that uses geographically and administratively distant resources, and, thus, users can access computers and data transparently without concern about location, operating system, and account administration" [11]. With the advent of cloud computing, the merits of virtualization and grid computing have been combined and further improved. Cloud computing shares some characteristics with virtualization and grid computing; however, it still has its own distinguishing characteristics as well as associated risks [12-15].

Cloud computing has been given numerous definitions since its advent. Basically, definitions started with the notion of an application service provision (ASP) that is an IT sourcing model for renting business applications over the Internet [16]. This definition became wider as Internet-based IT service offerings comprised storage, hosting infrastructure, and network; thus, it is given the name net sourcing, to fit the variety of IT service offerings [17]. HP defines cloud computing as "Everything as a Service" [18], while Microsoft perceives the value of cloud computing as "Cloud + Client," emphasizing the importance of the end user [19]. T-Systems define cloud computing as "the renting of infrastructure and software, as well as bandwidths, under defined service conditions. These components should be able to be adjusted daily to the needs of the customer and offered with the utmost availability and security. Included in cloud computing are end-2-end service level agreements (SLAs) and use-dependent service invoices" [20].

T-Systems' definition conveys the idea of cloud computing as being a utility computing or 5th utility, because enterprises are able to consume computing resources on a pay-as-you-go basis just like the four public utilities (water, electricity, gas, and telephone). The widely known definition of cloud computing is by the National Institute of Standards and Technology (NIST). The NIST defines cloud computing as "a model for enabling convenient, on-demand network access to a shared pool of configurable computing resources (e.g., networks, servers, storage, applications, and services) that can be rapidly provisioned and released with minimal management effort or service provider interaction" [21]. According to the NIST definition, the basic actors in the cloud computing context are the cloud service provider (CSP) and the cloud service consumer (CSC), despite that there might be service brokers involved [22].

CSPs offer various service models depending on the enterprise's requirements, whereas the basic service models are [21]: (1) Software-as-a-Service (SaaS), the capability of the consumer to use the provider's applications running on a cloud infrastructure; (2) Platform-as-a-Service (PaaS), the capability of the consumer to deploy onto the cloud infrastructure consumer-created or acquired applications created using programming languages, libraries, services, and tools supported by the provider; and (3) Infrastructure-as-a-Service (IaaS), the capability of the consumer to provision 
processing, storage, networks, and other fundamental computing resources, where the consumer is able to deploy and run arbitrary software, which can include operating systems and applications. It has been reported that $32 \%$ of large enterprises are testing the concept of cloud computing; 37\% are already running applications on the cloud; and $17 \%$ are using cloud infrastructure [7]. Contrarily, $19 \%$ of SMEs are testing the concept; $29 \%$ are running applications on the cloud; and $41 \%$ are using cloud infrastructure [7].

Cloud computing service models share five common essential characteristics that distinguish cloud computing from other computing technologies [21]:

1. On-demand self-service, where the consumer can unilaterally provision computing capabilities, such as server time and network storage, as needed automatically without requiring human interaction with each service provider;

2. Broad network access, where the capabilities are available over the network and accessed through standard mechanisms that promote use by heterogeneous thin or thick client platforms (e.g., mobile phones, tablets, laptops, and workstations);

3. Resource pooling, where the provider's computing resources are pooled to serve multiple consumers using a multitenant model, with different physical and virtual resources dynamically assigned and reassigned according to consumer demand;

4. Rapid elasticity, where capabilities can be elastically provisioned and released, in some cases automatically, to scale rapidly outward and inward commensurate with demand; and

5. Measured service, where cloud systems automatically control and optimize resource use by leveraging a metering capability at some level of abstraction appropriate to the type of service (e.g., storage, processing, bandwidth, and active user accounts).

Cloud service models can be deployed in one of the four deployment models [21]: (1) private cloud, where the cloud infrastructure is provisioned for exclusive use by a single organization comprising multiple consumers (e.g., business units), and it may be owned, managed, and operated by the organization, a third party, or some combination of them, and it may exist on or off premises; (2) community cloud, where the cloud infrastructure is provisioned for exclusive use by a specific community of consumers from organizations that have shared concerns (e.g., mission, security requirements, policy, and compliance considerations), and it may be owned, managed, and operated by one or more of the organizations in the community, a third party, or some combination of them, and it may exist on or off premises; (3) public cloud, where the cloud infrastructure is provisioned for open use by the general public. It may be owned, managed, and operated by a business, academic, or government organization, or some combination of them, and it exists on the premises of the cloud provider; (4) hybrid cloud, where the cloud infrastructure is a composition of two or more distinct cloud infrastructures (private, community, or public) that remain unique entities but are bound together by standardized or proprietary technology that enables data and application portability (e.g., cloud bursting for load balancing between clouds). It has been reported recently that $61 \%$ of enterprises are currently using public clouds; $38 \%$ are using private clouds; and $29 \%$ are using hybrid clouds [7]. 
At the enterprise level, cloud computing adoption takes place in three contexts. Large enterprises have slack resources, both financial and technical, to afford deploying private IaaS, PaaS, and SaaS clouds on a pay-as-you-go basis [4, 23]. Whereas, SMEs tend to deploy public SaaS clouds, which are appropriate for their start-up due to their limited financial and IT capabilities, which impede their deploying and maintaining private clouds [4, 24-26]. For governments, deployment of private IaaS clouds is favorable [4].

In spite of its appealing benefits for enterprises, cloud computing raises serious technical, economic, ethical, legal, and managerial issues $[6,27]$. The extant literature is focused more on the technical issues of cloud computing, with less attention paid to business issues regarding the adoption of cloud computing [10]. Further, there is a lack of in-depth studies about issues related to the cloud computing adoption process in the context of enterprise users $[10,28]$. The purpose of this study is to review systematically the extant literature regarding cloud computing adoption to identify the key issues that have been researched. In addition, the quality of the extant research will be assessed. Then, the under-researched areas will be identified, and a future IS research agenda will be proposed accordingly. The remainder of this study is organized as follows: Section 2 presents the methodology of the systematic literature search process and the classification schemes adopted. Section 3 presents the findings from the review. Implications for future IS research are discussed in Section 4. Conclusions of this review are presented in Section 5.

\section{$2 \quad$ Research Method}

Reviewing the literature is an essential process that creates a firm foundation for advancing knowledge; it facilitates uncovering areas where research is needed [29]. This paper aims at systematically reviewing the literature to represent the current state of IS research regarding cloud computing adoption issues. This review process followed the fundamental guidelines for conducting an effective literature review by [29-31], and it is done within boundaries [29]. The contextual boundary for this review is the enterprise users, not individuals, as there are significant issues that need to be addressed before enterprises start using clouds [27, 32]. The temporal boundary of this review covers the published articles in all previous years until February 2014.

\subsection{Literature Search Process}

The literature search process of this review involved querying seven quality scholarly literature databases (AISeL, IEEE Xplore, ScienceDirect, EBSCOhost, ProQuest, Wiley online library, and ACM digital library). These databases provide access to leading IS journals and high-quality peer-reviewed IS conference publications [31]. Further, online databases are appropriate and practical sources for reviewing the literature about a contemporary phenomenon such as cloud computing [10]. The search criterion was limited to the article's title to ensure the relevance of the articles. The terms used for searching all seven databases are 'cloud computing' in combination with 'adopt*' and other related terms, such as 'accept*' and 'diffus*'. This initially resulted in 94 articles in total including recurrences. An overview of the search process is provided in Table 1. 
Table 1. Literature search overview

\begin{tabular}{llc}
\hline $\begin{array}{l}\text { Literature data- } \\
\text { bases }\end{array}$ & Search query & $\begin{array}{c}\text { Search } \\
\text { results }\end{array}$ \\
\hline AISeL & title:"cloud computing" AND title:adopt* & 15 \\
& title:"cloud computing" AND title:accept* & 2 \\
& title:"cloud computing" AND title:diffus* & 0 \\
\hline IEEE Xplore & (("Document Title":"cloud computing") AND "Document & 22 \\
& Title":"adopt*") & 2 \\
& (("Document Title":"cloud computing") AND "Document & \\
& Title":"accept*") & 1 \\
& (("Document Title":"cloud computing") AND "Document & 6 \\
& Title":"diffus*") & 0 \\
\hline ScienceDirect & TITLE("cloud computing") and TITLE(adopt*) & 0 \\
\hline EBSCOhost & TITLE("cloud computing") and TITLE(accept*) & 30 \\
& TITLE("cloud computing") and TITLE(diffus*) & 0 \\
\hline ProQuest & TI "cloud computing" AND TI "adopt*" & 6 \\
& TI "cloud computing" AND TI "accept*" & 1 \\
& ti("cloud computing") AND ti(adopt*) & 0 \\
\hline Wiley (Online & ti("cloud computing") AND ti(accept*) & 1 \\
Library) & "cloud computing" in Article Titles AND "adopt*" in Article & 1 \\
& Titles & \\
\hline Total & "cloud computing" in Article Titles AND accept* in Article & 0 \\
\hline Library) & Titles & 0 \\
\hline & "cloud computing" in Article Titles AND diffus* in Article Titles & 0 \\
\hline & (Title:"cloud computing" and Title:"adopt*") & 5 \\
\hline & (Title:"cloud computing" and Title:"accept*") & 1 \\
\hline
\end{tabular}

The practical screen involved reading the abstract of the articles to decide their relevance to the focus of this review [30, 33]. Further, the filtering criteria involved the exclusion of recurring articles, research-in-progress articles, articles that were not written in English, articles with a focus on individuals, periodical articles published by news websites, trade journals, and magazines. These exclusion criteria delimit the sample of articles so that the literature review becomes practically manageable [33]. Eventually, this resulted in 51 articles for the classification.

\subsection{Classification Scheme}

The reviewed articles are classified according to the research methods employed in each study to identify how adequately the adoption of cloud computing is researched [34-36]. The research methods used in the reviewed articles are lab experiments, field studies, Delphi study, interviews, literature reviews, case studies, and surveys. Some articles do not have a methodology section and reflect on some concepts in relation to cloud computing (i.e., cost, security, performance, etc.) or they adopt theories without empirical testing. This group of articles is labeled as "conceptual papers."

Cloud computing adoption issues are discussed diversely in the literature; thus, this review sought to develop a classification scheme to better gain insights from the 
preceding academic contributions to the area of cloud computing adoption. The classification of the 51 articles involved using a bottom-up grounded theory (GT) approach [37]. The GT approach is said to be valuable for conducting a rigorous literature review [38], "instead of force-fitting the data to an a priori theory" [39]. The 51 reviewed articles are classified according to a GT approach for reviewing the literature recommended by [38]. Using a GT approach in reviewing the literature helps "reach a thorough and theoretically relevant analysis of a topic" [38].

The classification process involved a close reading of the articles. Then, the open coding was utilized to generate codes from analyzing each article's text to capture the themes that appear in each article. This resulted in 30 concepts, which were labeled. Next, the axial coding was applied to develop the relations between the concepts identified in the open coding [38]. This resulted in a grouping of the 30 subcategories identified from the open coding into eight corresponding categories (i.e., internal, external, evaluation, proof of concept, adoption decision, implementation and integration, IT governance, and confirmation).

Finally, the selective coding technique was applied to integrate and refine the eight main categories and to develop relations between them [38]. This resulted in two abstract categories: cloud adoption factors (i.e., internal and external) and cloud adoption processes (i.e., evaluation, proof of concept, adoption decision, implementation and integration, IT governance, and confirmation), where the former influenced the latter (see Table 2).

Table 2. Classification scheme

\begin{tabular}{|c|c|c|}
\hline Selective coding & Axial coding & Open coding \\
\hline \multirow[t]{2}{*}{$\begin{array}{l}\text { Cloud computing } \\
\text { adoption factors }\end{array}$} & 1. External & $\begin{array}{ll}\text { 1. } & \text { Government regulations } \\
\text { 2. } & \text { IT industry standards institutes } \\
\text { 3. } & \text { Cloud providers } \\
\text { 4. } & \text { Business partners } \\
\text { 5. } & \text { Competitors } \\
\text { 6. } & \text { Cloud service broker } \\
\end{array}$ \\
\hline & 2. Internal & $\begin{array}{ll}\text { 7. } & \text { Willingness to invest } \\
\text { 8. } & \text { Top management } \\
\text { 9. } & \text { Firm size } \\
\text { 10. } & \text { Organizational culture } \\
\text { 11. } & \text { Employees' IT skills } \\
\text { 12. } & \text { Prior experience } \\
\end{array}$ \\
\hline \multirow[t]{3}{*}{$\begin{array}{l}\text { 2. Cloud computing } \\
\text { adoption processes }\end{array}$} & 3. Evaluation & $\begin{array}{l}\text { 13. Cost and benefits } \\
\text { 14. Impact on people and work } \\
\text { practices } \\
\text { 15. Internal readiness } \\
\text { 16. Cloud provider selection }\end{array}$ \\
\hline & 4. Proof of concept & $\begin{array}{l}\text { 17. Trialability } \\
\text { 18. Perceived benefits and risks }\end{array}$ \\
\hline & Adoption decision & $\begin{array}{ll}19 . & \text { Business needs identification } \\
\text { 20. } & \text { Criticality determination } \\
\text { 21. } & \text { Strategic value evaluation } \\
\text { 22. Implementation planning } \\
\text { 23. Service model selection } \\
\text { 24. } \\
\text { 25. } & \text { Contract and SLA negotiation }\end{array}$ \\
\hline
\end{tabular}


Table 2. (Continued)

\begin{tabular}{llll}
\hline 6. & Implementation & 26. & Complexity \\
& and integration & 27. & Compatibility \\
\hline 7. & IT governance & $\begin{array}{l}\text { 28. } \\
\text { 29. }\end{array}$ Risk managitity and traceability \\
\hline 8. & Confirmation & 30. & Usage continuance \\
\hline
\end{tabular}

\section{$3 \quad$ Findings}

The findings from reviewing 51 articles are presented in light of six dimensions: distribution of articles over years, outlets in which articles were published, theories/frameworks used, research methods used, and cloud computing adoption factors and processes. Fig. 1 illustrates that interest in researching the topic of cloud computing adoption has grown exponentially from 2009 until 2013, denoting that cloud computing adoption is remarkable. However, the three articles published in 2014 do not present a full picture of research endeavors of the whole year 2014 .

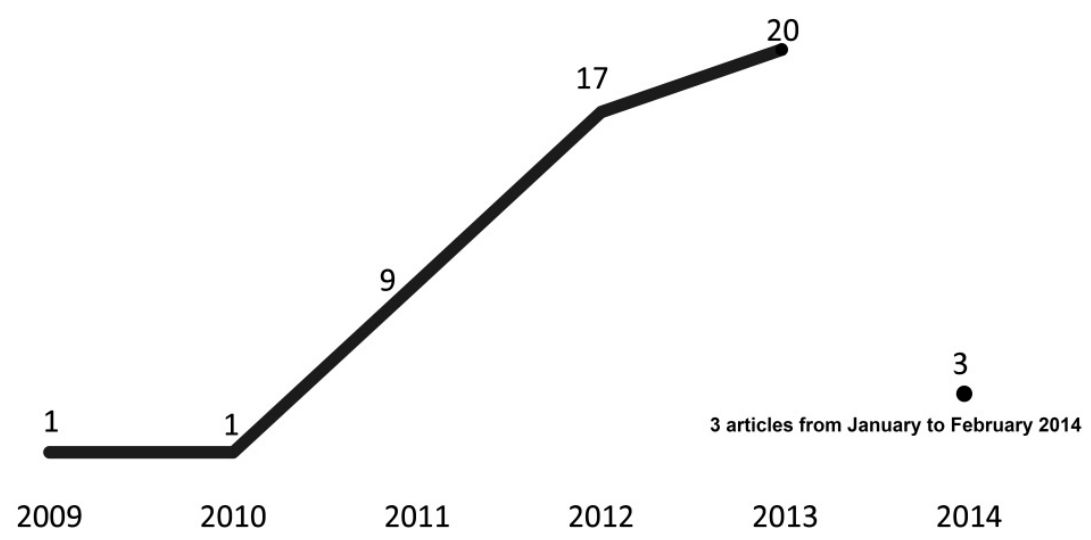

Fig. 1. Distribution of articles over years

The outlets in which the articles were published and the number of articles $(\mathrm{N})$ in each are presented in Table 3; the outlets are categorized as IS and non-IS journals and conferences. The IS journals and conferences are identified according to the Association for Information Systems (AIS) $)^{1,2,3,4}$ and [31]. Obviously, non-IS journals dominate in investigating cloud computing adoption area with 11 articles each, whereas only one IS journal contributed with three articles. Likewise, non-IS conferences contributed numerously, with 25 articles, whereas IS conferences had 12 articles. However, more published articles were found in IS conference publications than in IS journals.

\footnotetext{
${ }^{1}$ http: //aisnet.org/general/custom.asp?page=JournalRankings

${ }^{2}$ http://aisnet.org/?AffiliatedConference

${ }^{3}$ http: / / aisnet.org/ ?page=Conferences

${ }^{4}$ http://aisel.aisnet.org/affiliated/
} 
Table 3. Number of articles per outlet type

\begin{tabular}{|c|c|c|}
\hline Outlet type & Outlet name & $\mathrm{N}$ \\
\hline IS Journals & $\begin{array}{ll} & \text { International Journal of Information Management } \\
\end{array}$ & 3 \\
\hline $\begin{array}{l}\text { Non-IS } \\
\text { Journals }\end{array}$ & $\begin{array}{ll}\text { - } & \text { Journal of Technology Management for Growing Economies } \\
\text { - } & \text { Journal of Medical Marketing: Device, Diagnostic and Pharma- } \\
\text { - } & \text { Mathemal Marketing } \\
\text { - } & \text { Industrial Management \& Data Systems } \\
\text { - } & \text { International Journal of Logistics Management } \\
\text { - } & \text { Journal of Enterprise Information Management } \\
\text { - } & \text { IEEE Transactions on Engineering Management } \\
\text { - } & \text { European Management Review } \\
\text { - } & \text { Procedia Technology } \\
\text { - } & \text { Journal of Industry, Competition, and Trade } \\
\text { - } & \text { GSTF Journal on Computing }\end{array}$ & 11 \\
\hline $\begin{array}{l}\text { IS Confe- } \\
\text { rences }\end{array}$ & $\begin{array}{ll}\text { - } & \text { International Conference on Information Systems (ICIS) } \\
\text { - } & \text { Americas Conference on Information Systems (AMCIS) } \\
\text { - } & \text { Hawaii International Conference on System Sciences (HICSS) } \\
\text { - } & \text { Pacific Asia Conference on Information Systems (PACIS) } \\
\text { - } & \text { Mediterranean Conference on Information Systems (MCIS) }\end{array}$ & 12 \\
\hline $\begin{array}{l}\text { Non-IS } \\
\text { Conferences }\end{array}$ & 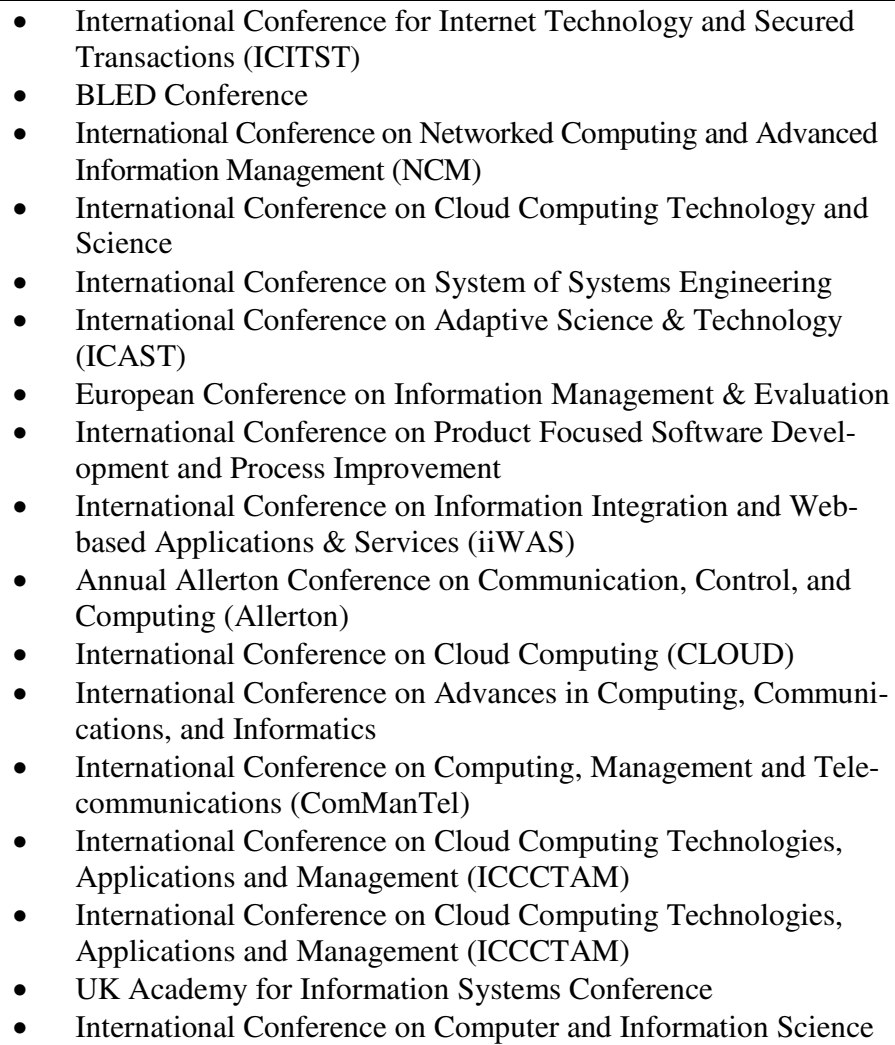 & 25 \\
\hline
\end{tabular}


Table 3. (Continued)

- $\quad$ IST-Africa Conference and Exhibition

- International Conference on Research and Innovation in Information Systems (ICRIIS)

- International Conference on ICT for Smart Society

- IEEE World Congress on Services

The findings from the 51 articles are further organized according to theory/ framework/model used in each publication. Some articles discuss related concepts (i.e., performance, cost, security, cloud providers, etc.); thus, they are considered to be using "general concepts." Further, other articles use a GT approach to understand cloud computing adoption; these articles built models based on field data. The use of different theories to understand cloud computing adoption factors and processes is quite evident, as per Table 4. Further, articles that tested theories empirically by field data are predominant, but the non-empirically tested theoretical contributions are not slight. General concepts are the most frequently used to explain cloud computing adoption factors and processes.

The use of the technology-organization-environment (TOE) framework is also frequent compared to other theories, followed by diffusion of innovation (DOI) theory and the GT approach, which appear to be the next most frequently used in the reviewed articles. Empirically tested theories/frameworks/models are dominant with 34 articles, whereas studies with no empirical testing were less common: 17 articles. However, the number of articles without empirical testing is still remarkable, which implies the need for more field work. Further, the majority of articles used a combination of multiple theoretical perspectives to gain more insights about cloud computing adoption factors and processes. This implies that cloud computing adoption phenomenon is multifaceted. The majority of articles have used theories/frameworks/models to explain what are the factors that affect the adoption of cloud computing and what are the key considerations in cloud computing adoption processes. Yet, there is a lack of using theories that demonstrate how enterprises react differently to same internal and external factors and why do they react in such a way.

Table 4. Use of theory by reviewed articles

\begin{tabular}{llllc}
\hline Theory (T)/Framework(F)/Model(M) & $\begin{array}{l}\text { References } \\
\text { (Empirical } \\
\text { Testing) }\end{array}$ & $\begin{array}{c}\text { References (no } \\
\text { Empirical Testing) }\end{array}$ & Frequency \\
\hline (M) & $\begin{array}{l}\text { Technology Acceptance } \\
\text { Model (TAM) }\end{array}$ & {$[40],[41],[42]$} & - & 3 \\
\hline (M) & $\begin{array}{l}\text { Post-Acceptance Model } \\
\text { (PAM) of IS Continuance }\end{array}$ & {$[43]$} & - & 1 \\
\hline (T) & Utility Theory & {$[44]$} & - & 1 \\
\hline (M) & $\begin{array}{l}\text { Human-Organization- } \\
\text { Technology Fit Model (HOT- } \\
\text { fit) }\end{array}$ & {$[45]$} & - & 1 \\
\hline (M) & Business Model Factors & {$[46]$} & - & 1 \\
\hline (M) & $\begin{array}{l}\text { Structural Equation Modeling } \\
\text { (SEM) }\end{array}$ & {$[47]$} & - & 1 \\
\hline
\end{tabular}


Table 4. (Continued)

\begin{tabular}{|c|c|c|c|c|}
\hline (F) & $\begin{array}{l}\text { Technology-Organization- } \\
\text { Environment (TOE) }\end{array}$ & $\begin{array}{l}\text { [48], [49], [50], } \\
{[51],[52],[45],} \\
{[53],[54],[55]}\end{array}$ & {$[56],[23]$} & 11 \\
\hline (T) & $\begin{array}{l}\text { Organizational Information } \\
\text { Processing Theory }\end{array}$ & {$[57]$} & - & 1 \\
\hline- & General Concepts & $\begin{array}{l}58],[59],[60], \\
{[61],[62],[63]}\end{array}$ & $\begin{array}{l}64],[65],[66], \\
{[67],[68],[69],} \\
{[70],[71],[72]} \\
\end{array}$ & 15 \\
\hline (F) & $\begin{array}{l}\text { National Outsourcing Associ- } \\
\text { ation (NOA) Framework for } \\
\text { Factors Inhibiting Cloud } \\
\text { Computing Adoption }\end{array}$ & [73] & - & 1 \\
\hline (F) & $\begin{array}{l}\text { Contextual Usability Frame- } \\
\text { work }\end{array}$ & [74] & - & 1 \\
\hline (F) & Attention Based View (ABV) & {$[75]$} & - & 1 \\
\hline (T) & $\begin{array}{l}\text { Diffusion Of Innovation } \\
\text { (DOI) }\end{array}$ & [76], [40], [74] & [56], [23], [77] & 6 \\
\hline (T) & $\begin{array}{l}\text { Transaction Cost Economics } \\
\text { (TCE) Theory }\end{array}$ & - & {$[78],[77]$} & 2 \\
\hline (T) & $\begin{array}{l}\text { Resource Dependence Theory } \\
\text { (RDT) }\end{array}$ & - & [77] & 1 \\
\hline (T) & $\begin{array}{l}\text { Innovation Decision Process } \\
\text { Theory (IDPT) }\end{array}$ & - & [79] & 1 \\
\hline$(\mathbf{T})$ & Dynamic Capabilities Theory & [40] & - & 1 \\
\hline (T) & Contingency Theory & [40] & - & 1 \\
\hline (T) & $\begin{array}{l}\text { Mean Field Game Theory } \\
\text { (MFG) }\end{array}$ & - & {$[80]$} & 1 \\
\hline (M) & Return On Investment (ROI) & - & {$[81]$} & 1 \\
\hline (T) & Option Pricing Theory & - & {$[82]$} & 1 \\
\hline (T) & Perceived Attributes Theory & - & [79] & 1 \\
\hline (F) & $\begin{array}{l}\text { Geoffrey Moore's Technolo- } \\
\text { gy Adoption Life Cycle }\end{array}$ & - & [79] & 1 \\
\hline (F) & $\begin{array}{l}\text { Innovation Value Institute's } \\
\text { IT Capability Maturity } \\
\text { Framework (ITCMF) }\end{array}$ & [83] & - & 1 \\
\hline$(\mathbf{T})$ & Actor Network Theory (ANT) & {$[48],[55]$} & - & 2 \\
\hline (M) & Gap Analysis Model & [84] & - & 1 \\
\hline (T) & GT Approach & $\begin{array}{l}\text { [85], [86], [87], } \\
{[88],[89]}\end{array}$ & - & 5 \\
\hline \multicolumn{2}{|c|}{ Total (without repetitions) } & 34 & 17 & \\
\hline
\end{tabular}

As per Table 5, the 51 articles are mapped to cloud computing adoption factors (i.e., external and internal) and processes (i.e., evaluation, proof of concept, adoption decision, implementation and integration, IT governance, and confirmation) identified in this review as well as research methods (RM) employed (i.e., Lab Experiment (LE), Field Study (FS), Case Study (CS), Delphi Study (DS), Survey (SUR), Interviews (INT), Conceptual Paper (CP), Literature Review (LR)). Further, the number of articles $(\mathrm{N})$ per subcategory and research method is provided. The findings, in general, 
indicate fewer qualitative studies (i.e., case studies, interviews, and field studies) have contributed to the understanding of cloud computing adoption factors and processes, as compared to quantitative studies (i.e., surveys). In some articles, multiple methods are used [42, 44, 50, 57]. Further, external adoption factors are extensively addressed by survey and conceptual articles, and less addressed by in-depth studies. This applies similarly to the internal factors. Among the external factors, investigating government regulations is dominant. Regarding the internal factors, the role of top management in cloud computing adoption is more researched among others. In general, adoption processes, such as evaluation, adoption decision, implementation and integration, IT governance, and confirmation, are not adequately addressed, except for the proof of concept process. However, the number of studies identifying perceived benefits and risks is predominant in proof of concept process and among other cloud computing adoption processes.

Table 5. Mapping of articles to classification scheme and research methods

\begin{tabular}{|c|c|c|c|c|c|c|c|c|c|}
\hline \multicolumn{2}{|c|}{$\begin{array}{l}\text { Cloud computing adoption } \\
\text { factors and processes }\end{array}$} & \multirow{2}{*}{$\begin{array}{l}\text { LE } \\
-\end{array}$} & \multirow{2}{*}{$\begin{array}{l}\text { FS } \\
{[61]}\end{array}$} & \multirow{2}{*}{$\begin{array}{l}\text { CS } \\
{[83],} \\
{[48],} \\
{[89],} \\
{[84]}\end{array}$} & \multirow{2}{*}{$\begin{array}{l}\text { DS } \\
{[57],} \\
{[87]}\end{array}$} & \multirow{2}{*}{$\begin{array}{l}\text { SUR } \\
{[57],} \\
{[73],} \\
{[54],} \\
{[50],} \\
{[55],} \\
{[45]}\end{array}$} & \multirow{2}{*}{$\begin{array}{l}\text { INT } \\
{[57],} \\
{[50]}\end{array}$} & \multirow{2}{*}{$\begin{array}{l}\mathrm{CP} \\
{[23],} \\
{[77],} \\
{[69],} \\
{[78],} \\
{[68],} \\
{[79],} \\
{[72],} \\
{[70]}\end{array}$} & \multirow{2}{*}{$\begin{array}{l}\text { LR } \\
-\end{array}$} \\
\hline $\begin{array}{l}\text { External } \\
\text { factors }\end{array}$ & $\begin{array}{l}\text { Government } \\
\text { regulations } \\
(\mathrm{N}=20)\end{array}$ & & & & & & & & \\
\hline & $\begin{array}{l}\text { IT industry } \\
\text { standards } \\
\text { institutes } \\
(\mathrm{N}=6)\end{array}$ & - & - & [83] & - & $\begin{array}{l}76], \\
{[54]}\end{array}$ & - & $\begin{array}{l}\text { [77], } \\
{[78],} \\
{[71]}\end{array}$ & - \\
\hline & $\begin{array}{l}\text { Cloud pro- } \\
\text { viders } \\
(\mathrm{N}=15)\end{array}$ & - & - & $\begin{array}{l}{[51],} \\
{[48],} \\
{[89]}\end{array}$ & - & $\begin{array}{l}{[75],} \\
{[86],} \\
{[76],} \\
{[85],} \\
{[55],} \\
{[53],} \\
{[46]}\end{array}$ & - & $\begin{array}{l}{[78],} \\
{[79],} \\
{[68],} \\
{[77],} \\
{[72]}\end{array}$ & - \\
\hline & $\begin{array}{l}\text { Business } \\
\text { partners } \\
(\mathrm{N}=11)\end{array}$ & - & - & [48] & [57] & $\begin{array}{l}{[52],} \\
{[76],} \\
{[57],} \\
{[75],} \\
{[85],} \\
{[85],} \\
{[55],} \\
{[74],} \\
{[42],} \\
{[53]}\end{array}$ & $\begin{array}{l}{[57],} \\
{[42]}\end{array}$ & [56] & - \\
\hline & $\begin{array}{l}\text { Competitors } \\
(\mathrm{N}=10)\end{array}$ & - & - & $\begin{array}{l}{[51],} \\
{[48]}\end{array}$ & [57] & $\begin{array}{l}{[57],} \\
{[52],} \\
{[50],} \\
{[55],} \\
{[53],} \\
{[45]}\end{array}$ & $\begin{array}{l}{[57],} \\
{[50]}\end{array}$ & $\begin{array}{l}{[23],} \\
{[56]}\end{array}$ & - \\
\hline
\end{tabular}


Table 5. (Continued)

\begin{tabular}{|c|c|c|c|c|c|c|c|c|c|}
\hline & $\begin{array}{l}\text { Cloud service } \\
\text { broker } \\
(\mathrm{N}=1)\end{array}$ & - & - & - & - & - & - & [71] & - \\
\hline \multirow[t]{6}{*}{$\begin{array}{l}\text { Internal } \\
\text { factors }\end{array}$} & $\begin{array}{l}\text { Willingness } \\
\text { to invest } \\
(\mathrm{N}=11)\end{array}$ & - & - & $\begin{array}{l}83], \\
{[40],} \\
{[48],} \\
{[51]}\end{array}$ & - & $\begin{array}{l}60], \\
{[76],} \\
{[50],} \\
{[44],} \\
{[41]}\end{array}$ & $\begin{array}{l}50], \\
{[44]}\end{array}$ & $\begin{array}{l}{[23],} \\
{[56]}\end{array}$ & - \\
\hline & $\begin{array}{l}\text { Top man- } \\
\text { agement } \\
(\mathrm{N}=18)\end{array}$ & - & [88] & $\begin{array}{l}40], \\
{[51],} \\
{[83],} \\
{[49]}\end{array}$ & [87] & $\begin{array}{l}{[50],} \\
{[73],} \\
{[60],} \\
{[55],} \\
{[74],} \\
{[53],} \\
{[45]}\end{array}$ & [50] & $\begin{array}{l}67], \\
{[23],} \\
{[81],} \\
{[64]}\end{array}$ & - \\
\hline & $\begin{array}{l}\text { Firm size } \\
(N=9)\end{array}$ & - & - & - & - & $\begin{array}{l}{[52],} \\
{[50],} \\
{[60],} \\
{[55],} \\
{[53]}\end{array}$ & [50] & $\begin{array}{l}{[23],} \\
{[56],} \\
{[81],} \\
{[72]}\end{array}$ & - \\
\hline & $\begin{array}{l}\text { Organization- } \\
\text { al culture } \\
(\mathrm{N}=5)\end{array}$ & - & - & $\begin{array}{l}499], \\
{[83]}\end{array}$ & - & $\begin{array}{l}{[50],} \\
{[47]}\end{array}$ & {$[50]$} & \begin{tabular}{l|l}
$71]$ \\
\end{tabular} & - \\
\hline & $\begin{array}{l}\text { Employees' } \\
\text { IT skills } \\
(\mathrm{N}=8)\end{array}$ & - & - & [49] & - & $\begin{array}{l}60], \\
{[50],} \\
{[52],} \\
{[55],} \\
{[45]}\end{array}$ & [50] & $\begin{array}{l}{[23],} \\
{[70]}\end{array}$ & - \\
\hline & $\begin{array}{l}\text { Prior expe- } \\
\text { rience } \\
(\mathrm{N}=6)\end{array}$ & - & - & [51] & - & $\begin{array}{l}{[41],} \\
{[74],} \\
{[42],} \\
{[53]}\end{array}$ & {$[42]$} & - & - \\
\hline \multirow[t]{4}{*}{$\begin{array}{l}\text { Evalua- } \\
\text { tion } \\
\text { process }\end{array}$} & $\begin{array}{l}\text { Cost and } \\
\text { benefits } \\
(\mathrm{N}=10)\end{array}$ & - & [61] & $\begin{array}{l}{[89],} \\
{[84]}\end{array}$ & & $\begin{array}{l}{[45],} \\
{[46]}\end{array}$ & - & $\begin{array}{l}{[81],} \\
{[80],} \\
{[79],} \\
{[82],} \\
{[70]}\end{array}$ & - \\
\hline & $\begin{array}{l}\text { Impact on } \\
\text { people and } \\
\text { work practices } \\
(\mathrm{N}=7)\end{array}$ & - & [61] & $\begin{array}{l}{[83],} \\
{[89]}\end{array}$ & - & $\begin{array}{l}42], \\
{[41]}\end{array}$ & [42] & $\begin{array}{l}{[70],} \\
{[71]}\end{array}$ & - \\
\hline & $\begin{array}{l}\text { Internal readi- } \\
\text { ness } \\
(\mathrm{N}=7)\end{array}$ & - & $\begin{array}{l}{[88]} \\
\end{array}$ & [84] & - & $\begin{array}{l}76], \\
{[52],} \\
{[42],} \\
{[45]}\end{array}$ & [42] & $\begin{array}{l}\text { [23] } \\
\end{array}$ & - \\
\hline & $\begin{array}{l}\text { Cloud provid- } \\
\text { er selection } \\
(\mathrm{N}=6)\end{array}$ & - & [61] & $\begin{array}{l}40], \\
{[83],} \\
{[84]}\end{array}$ & - & - & - & $\begin{array}{l}65], \\
{[67]}\end{array}$ & - \\
\hline $\begin{array}{l}\text { Proof of } \\
\text { concept } \\
\text { process }\end{array}$ & $\begin{array}{l}\text { Trialability } \\
(\mathrm{N}=4)\end{array}$ & - & - & [51] & - & $\begin{array}{l}{[76],} \\
{[74],} \\
{[53]}\end{array}$ & - & - & - \\
\hline
\end{tabular}


Table 5. (Continued)

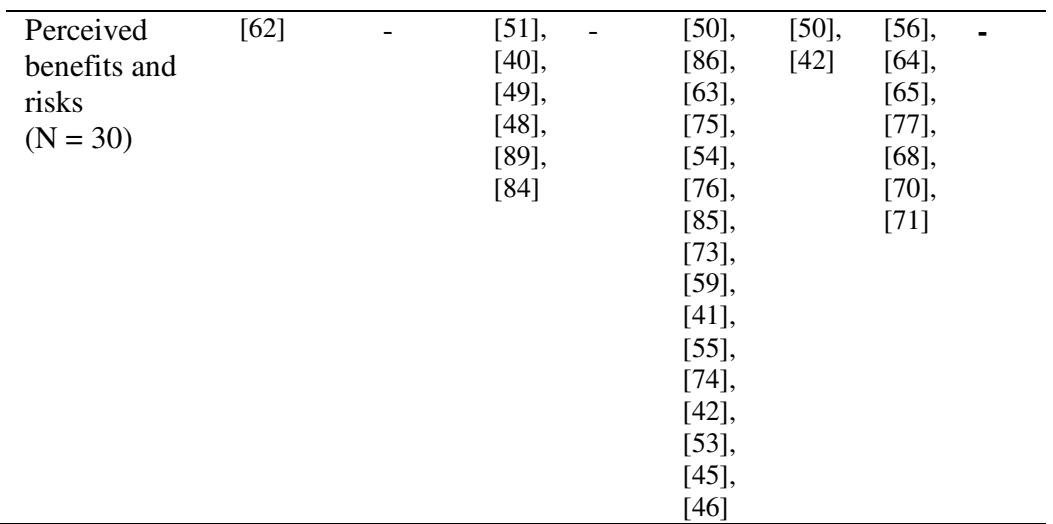

\begin{tabular}{|c|c|c|c|c|c|c|c|c|c|}
\hline \multirow[t]{7}{*}{$\begin{array}{l}\text { Adoption } \\
\text { decision } \\
\text { process }\end{array}$} & $\begin{array}{l}\text { Business } \\
\text { needs identi- } \\
\text { fication } \\
(\mathrm{N}=4)\end{array}$ & - & [61] & - & - & $\begin{array}{l}{[76],} \\
{[53]}\end{array}$ & - & [64] & - \\
\hline & $\begin{array}{l}\text { Criticality } \\
\text { determination } \\
(\mathrm{N}=3)\end{array}$ & - & - & - & - & - & - & $\begin{array}{l}{[69],} \\
{[81],} \\
{[82]}\end{array}$ & - \\
\hline & $\begin{array}{l}\text { Strategic value } \\
\text { evaluation } \\
(\mathrm{N}=3)\end{array}$ & - & - & [89] & - & $\begin{array}{l}75], \\
{[76]}\end{array}$ & - & - & - \\
\hline & $\begin{array}{l}\text { Implementa- } \\
\text { tion planning } \\
(\mathrm{N}=2)\end{array}$ & - & - & - & - & [60] & - & [79] & - \\
\hline & $\begin{array}{l}\text { Service mod- } \\
\text { el selection } \\
(\mathrm{N}=2)\end{array}$ & - & - & - & - & - & - & [65] & [66] \\
\hline & $\begin{array}{l}\text { Deployment } \\
\text { model selec- } \\
\text { tion } \\
(\mathrm{N}=2)\end{array}$ & - & - & - & - & - & - & $\begin{array}{l}\text { [79], } \\
{[69]}\end{array}$ & - \\
\hline & $\begin{array}{l}\text { Contract and } \\
\text { SLA negotia- } \\
\text { tion } \\
(\mathrm{N}=4)\end{array}$ & - & - & - & - & [53] & - & $\begin{array}{l}65], \\
{[81],} \\
{[71]}\end{array}$ & - \\
\hline \multirow[t]{2}{*}{$\begin{array}{l}\text { Imple- } \\
\text { mentation } \\
\text { and inte- } \\
\text { gration } \\
\text { process }\end{array}$} & $\begin{array}{l}\text { Complexity } \\
(\mathrm{N}=10)\end{array}$ & - & - & $\begin{array}{l}{[49],} \\
{[51]}\end{array}$ & - & $\begin{array}{l}{[50],} \\
{[76],} \\
{[52],} \\
{[74],} \\
{[42],} \\
{[53],} \\
{[45]}\end{array}$ & $\begin{array}{l}{[50],} \\
{[42]}\end{array}$ & {$[23]$} & - \\
\hline & $\begin{array}{l}\text { Compatibility } \\
(\mathrm{N}=9)\end{array}$ & - & - & {$[51]$} & - & $\begin{array}{l}{[50],} \\
{[76],} \\
{[52],} \\
{[74],} \\
{[53],} \\
{[45]}\end{array}$ & {$[50]$} & $\begin{array}{l}{[23],} \\
{[70]}\end{array}$ & - \\
\hline
\end{tabular}


Table 5. (Continued)

\begin{tabular}{|c|c|c|c|c|c|c|c|c|c|}
\hline \multirow[t]{2}{*}{$\begin{array}{l}\text { IT gover- } \\
\text { nance } \\
\text { process }\end{array}$} & $\begin{array}{l}\text { Auditability } \\
\text { and traceabil- } \\
\text { ity } \\
(\mathrm{N}=6)\end{array}$ & - & - & $\begin{array}{l}\text { [49], } \\
{[83]}\end{array}$ & - & [50] & {$[50]$} & $\begin{array}{l}65], \\
{[81],} \\
{[71]}\end{array}$ & - \\
\hline & $\begin{array}{l}\text { Risk man- } \\
\text { agement } \\
(\mathrm{N}=5)\end{array}$ & [62] & - & - & - & [63] & - & $\begin{array}{l}{[69],} \\
{[71]}\end{array}$ & [66] \\
\hline $\begin{array}{l}\text { Confir- } \\
\text { mation } \\
\text { process }\end{array}$ & $\begin{array}{l}\text { Usage conti- } \\
\text { nuance } \\
(\mathrm{N}=2)\end{array}$ & - & - & - & - & [43] & - & [79] & - \\
\hline $\mathrm{N}$ per $\mathrm{RM}$ & & 1 & 2 & 6 & 2 & 24 & 4 & 17 & 1 \\
\hline
\end{tabular}

Cloud Computing Adoption Factors. These comprise internal and external factors that have impact on the cloud computing adoption processes.

External Factors. These comprise factors from the outside social environment in which the enterprise operates and by which its cloud computing adoption process is influenced. These external factors are: 1) government regulations, 2) IT industry standards institutes, 3) cloud providers, 4) business partners, 5) competitors, and 6) cloud service broker. The adoption of cloud computing is arguably surrounded by different levels of environmental and inter-organizational uncertainties [57]. There is a wide emphasis on the importance of government regulations at the national and international levels $[23,54,73,77]$. In spite of their role in facilitating the cloud computing adoption securely, the lack of government regulations can hinder enterprises from adopting the cloud [45, 54, 87]. Some regulations, such as the Sarbanes-Oxley Act (SOX) for corporate accounting data, the Gramm-Leach-Bliley Act (GLBA), and the Health Insurance Portability and Accountability Act (HIPAA) were enacted before cloud computing was becoming increasingly adopted, and they might not be sufficient to facilitate its adoption [69, 72]. However, some countries started to enact laws specific to cloud computing, such as cloud first policy and the Health Information Technology for Economic and Clinical Health (HITECH) Act in the USA and cloud computing strategy by the Australian government $[72,78]$.

The inconsistency of international government regulations is a further concern as there is no widely agreed data privacy policy among all governments [72]. Some countries tend to restrict enterprises to store their data in cloud infrastructure only if it is within the national borders [68]. For instance, the EU's privacy laws prohibit the exchange of personal information outside the users' jurisdiction [83]. This is because cloud providers running outside of their home country must comply with the host country's regulations and government surveillance, which can be difficult for enterprises to cope with $[61,78]$. An example of the multijurisdictional politics that have negative impact on cloud computing adoption is the USA Patriot Act, which makes countries, especially, the EU countries, skeptical about dealing with US-based cloud providers [70, 72]. However, some cloud providers solved this issue to accord with EU data regulations by allowing enterprises to deploy their IT resources on physical servers located within the EU region [70]. Although government regulations are in place in the developed world, they often conflict with each other, and they are not adequately placed in the developing world $[72,78]$. 
In line with this, IT standards institutes are also cited as an important factor for dealing with enterprises' concerns about security and the interoperability of cloud solutions [77, 83]. Although they are still missing pieces in the puzzle of cloud computing for enterprises $[54,76,78]$, some efforts to develop security standards have occurred; for instance, Cloud Security Alliance (CSA)'s document Security Guidance for Critical Areas of Focus in Cloud Computing for managing cloud computing risks [83]. Cloud providers would have to comply with government regulations and industrial standards to gain liability, reputation, and trustworthiness among their potential and present enterprise users [78]. Additionally, they are required to be transparent in explaining information to enterprises about possible benefits and risks from adopting cloud computing [79]. Cloud providers build their reputations by their experience in managing enterprises' needs and concerns in a responsible manner [68, 75, 77]. This is enabled by delivering the promised benefits from cloud computing to enterprises (i.e., service quality, service availability, and service recovery) and protecting their data from potential threats; for instance, in case the cloud provider went out of business $[46,77,86]$. Further, cloud providers' demonstration of successful business cases and models are likely to increase cloud computing adoption rates [51, 76]. Moreover, the support for implementing and using cloud services made available by cloud services providers is likely to motivate enterprises to adopt cloud computing [53]. however, cloud providers might trigger a major concern for enterprises if they outsource some of their services to another service provider [77]. In this regard, trust issues are not well-explored yet.

Observing that business partners perceive benefits from using cloud computing has proven to be an important motive toward its adoption $[52,76]$. Another effect of business partners is that they may require an enterprise to adopt cloud computing if they want to remain in collaboration with them [57]. Additionally, competitors play an effective role in incentivizing enterprises to adopt cloud computing for gaining market visibility, operation efficiency, and new business opportunities [23, 50]. This especially happens when the enterprise operates in a high-tech, rapidly changing industry $[51,52]$. However, competitor pressure may not be relevant to SMEs, as they are more concerned about other cloud computing adoption issues such as cost reduction [51]. There is a lack of studies that explore the important role of the cloud service broker or the so-called service integrator in facilitating cloud computing adoption, with only one conceptual article, which described the G-Cloud program initiative in the UK. G-Cloud Authority is an internal cloud service broker that coordinates a managed and assured e-marketplace CloudStore of cloud services available to public sector organizations [71]. G-Cloud Authority eliminates the overhead for both cloud providers and consumers; the service makes it easy for cloud providers to sell cloud services, and enterprises do not need to spend a great deal of time in evaluating and selecting cloud providers [71]. The cloud service broker's role is to achieve a predictable end-to-end service outcome for enterprise users; this includes using standards for service management to predict, measure, and sustain cloud service outcomes [71]. Service management tools for cloud service brokers are available through the IT Infrastructure Library (ITIL); however, the ITIL is not mature enough yet [71]. The GCloud authority is responsible for providing cloud services once bought from the CloudStore, instantiating cloud services with appropriate business data, integrating the cloud service's management tools into the buying organization's service management 
framework, and billing coordination [71]. Being commissioned by industry associations enhances the cloud-service brokerage's trustworthiness [71].

Internal Factors. These comprise the enterprise's internal characteristics and capabilities that affect its cloud computing adoption processes; these factors are: (1) willingness to invest, (2) top management, (3) firm size, (4) organizational culture, and (5) employees' IT skills. The enterprise's willingness to invest in and use cloud computing both financially and organizationally is claimed to be an important indicator for the adoption of cloud computing [23, 60]. Willingness can be affected by social influences (i.e., subjective norms and image), as enterprises would adopt cloud computing because its managers said cloud computing is a good thing and can enhance the enterprise's status among its social system [41]. Cloud computing adoption is also dependent on the role of the top management, as there is a relationship between top management innovativeness (i.e., adopt and accept new technologies) and the willingness to adopt cloud computing [45, 53, 88]. Top management's IT knowledge, competence, and capability of providing the suitable organizational climate for adopting cloud computing in terms of budget, adequate human and IT resources, and time $[40,45,50,51]$ is a cornerstone to the adoption of cloud computing. This involves: (1) understanding of cloud computing and its architecture, service models, and strategic values [23, 60, 67, 73, 81, 87]; (2) identifying enterprise's business needs and aligning IT decisions with business strategies [64, 83]; (3) evaluating the readiness of the existing IT infrastructure, IT knowledge, and skills of the human resources, available resources, and culture [23, 50, 60, 87]; and (4) holding the steering wheel toward cloud computing adoption (i.e., decide on adoption strategy, govern integration and implementation, and evaluate cloud services after use) with the guidance of external regulatory and professional bodies $[49,83]$.

Additionally, firm size is an important factor to cloud computing adoption [23, 50, $52,56]$. A study claims that if the company is spread over many countries, then it is likely controlling its own IT resource and does not need to adopt cloud services [81]. Further, a survey study conducted in Taiwan indicated that large enterprises are likely to adopt cloud computing [52]. On the contrary, a survey study in India indicated that SMEs can benefit the most [60], because large enterprises have sufficient resources to afford on premise solutions [50]. Another survey study reported that large enterprises are likely to proof concept of cloud computing services, and that SMEs can be more flexible in adopting cloud computing [53]. A conceptual article stated that SMEs are likely to be price-oriented and less concerned about performance, whereas large enterprises tend to balance costs against reliability, security, and performance [72].

Furthermore, Organizational culture is said to have an impact on the enterprise's adoption of cloud computing [49]. For enterprises that were used to the on premise approach and having full control over their data, it might be difficult for them to accept that the cloud provider will be fully controlling their data. Thus, enterprises would need to further ensure compliance of cloud computing solutions with the internal (i.e., corporate policies) and external (i.e., regulations and standards) constraints $[49,50,83]$. Therefore, culture, capabilities, and processes can be barriers to the realization of cloud computing benefits; thus, cloud service brokers can assist enterprises to overcome these barriers [71]. A survey study advocates the need to identify the way of thinking of organizational elements (i.e., staff, and management) regarding 
culture that should be adjusted to meet the environmental needs and challenges in the future [47]. Further, employees' IT skills, especially non-IT employees', are said to be a crucial factor affecting cloud computing adoption decision, as their understanding of cloud computing is very important $[23,50,52,60]$. Likewise, IT employees would have to adjust their skills to be able to use cloud solutions [49]. Another factor affecting cloud computing adoption decision is the enterprise's prior experience and familiarity with similar technologies, such as virtualization [51].

Cloud Computing Adoption Processes. These comprise processes that enterprises normally follow to adopt cloud computing along with the responsibilities and challenges faced in each process:

Evaluation. This comprises (1) costs and benefits, (2) impact on people and work practices, (3) internal readiness, and (4) cloud provider selection. Prior to cloud computing adoption, the top management is responsible for evaluating the enterprise's suitability for adopting cloud computing as well as the suitability of cloud computing for the enterprise [76, 81]. This includes: evaluating the costs and benefits associated with cloud computing in the long and short term, such as profitability, comparing the revenue generated from the firm's IT resources with the revenue expected from cloud computing, ROI, cost of migration and integration, cost of implementation, and hidden costs, such as support and disaster recovery [70, 80, 81]. Evaluating the impact of cloud computing on people and work practices is also a must [83], as it may change the role of IT staff and require them to acquire new skill sets (i.e., some jobs may be merged). Regarding the impact, chief information officers (CIOs) may feel they are at risk of losing relevance and, to overcome this, CIOs will need to contribute to business strategy and information management [71], which requires a change in culture and skills across the enterprise led by CIOs [71]. Further, cloud computing is argued to have a job relevance impact, that is, the extent to which cloud computing enhances the enterprise's status and day-to-day operations and provides services applicable to employees' jobs so that they have control over their work and complete their tasks quickly [42].

Evaluating the internal readiness of the enterprise, existing IT infrastructure and IT human resources, for adopting cloud computing [52] - in terms of having sufficient and reliable resources to support the use of cloud computing as well as appropriate learning routines and performance measures is argued to enable the adoption of cloud computing $[42,88]$. Selecting the cloud provider based on the cloud provider's capability to provide robust security controls, the enterprise's understanding of issues related to the control over the data, the type of service model needed, and the perceived cloud provider's honesty, reputation, and sustainability [40, 65].

Proof of Concept. This comprises (1) trialability and (2) perceiving benefits and risks. Trialability is found to have a positive impact on the adoption of cloud computing [51]. Trying cloud services prior to the actual adoption to evaluate its applicability for the enterprise is likely to convince the enterprise to adopt cloud computing [76]. In this process, convincing enterprises to adopt cloud computing can be influenced by how they perceive cloud services. There is a wide agreement on the significant influence of the perceived benefits and risks on the adoption of cloud services [51,86]. 
This is relatively in line with a survey study's findings that the management's perceptions of security, cost-effectiveness, and IT compliance are likely to have a significant impact on the decision to adopt cloud computing [63]. Cloud computing brings plenty of benefits that are relatively convincing for enterprises to adopt it. This includes cost savings, agility, flexibility, ease of use, scalability, facilitating collaboration between business partners, less operational effort on CIOs, and increased productivity [40, 50, $54,64,75,76,85]$.

Even with all these enticing benefits, some SMEs are still negative about adopting cloud computing services [51]. SMEs are concerned with various types of risks [54, $65,68,70,76,77,86]$ :

- Organizational risks, which cover the risk of vendor lock-in as well as the loss of governance within the enterprise.

- Technical risks, which include data leakage, loss of data, downtime, data bottlenecks, and cyber-attacks.

- Legal risks, which include data protection regulations and licensing issues.

- Nontechnical risks, which refer to the misuse of cloud services and natural disasters.

- Performance risks, which are primarily that the moving of huge amounts of data to cloud servers takes a long time, and when moving further in the adoption, this will require increasing bandwidth and connectivity, which is costly [49].

Thus, benefits and risks perceived from trying cloud services will help enterprises to decide whether to adopt or not to adopt cloud computing.

Adoption Decision. This process comprises: (1) identifying business needs, (2) determining criticality, (3) evaluating strategic value, (4) implementation planning, (5) selecting the service model, (6) deploying model selection, and (7) contracting and SLA negotiation. When deciding to adopt cloud computing services, the top management is involved in the following activities: evaluating core business needs and competencies (i.e., quick response to market changes and increasing productivity) [61, 76], determining criticality in terms of what data and applications should move to the cloud (i.e., critical vs. noncritical data and applications) [69, 81, 82], evaluating the strategic value that cloud computing might bring to the enterprise, such as agility by delivering strong coordination IT capabilities, process management maturity, and reduced operational burden on CIOs so they can focus on strategic activities [75], planning for implementation of cloud computing systems in terms of the managerial time required to plan and implement cloud solutions was not problematic [60], whereas the problem was the planning for implementation of specific deployment models that suit the current applications [79]).

Furthermore, adoption decision process involves selecting the right service model (i.e., SaaS, PaaS, IaaS, or combined choice) that best fits the enterprise's needs [65, 66], selecting deployment models based on the sensitivity of the data and applications, if the data and applications are determined to be core, then they should be deployed on a private cloud, and if the applications are determined to be noncore, then they should be deployed on a public cloud [79], whereas another study suggests the core data and applications should not be deployed on the cloud at all [69]. Finally, the adoption decision is dependent on negotiating the cloud service contract and SLA 
with the cloud provider, based on the sensitivity of the data [81], and reaching an agreement on $[62,65,70,71]$ : the modifications to the contract terms, description of services (cost, price, and service content), limitation on the use or reuse of the data, which includes the data sanitization policy to ensure that data are securely removed when the use of cloud services ceases, confidentiality and security requirements in terms of organizational standards for data encryption both at rest and in flight, risk management plans, indemnification, contract terms and renewal, effect of termination, ownership of the data and applications, location of the data, assurance of service availability and expected downtimes, employees access control and protective monitoring, and clarity on roles and responsibilities. These items should be discussed with the cloud provider before proceeding to implementation.

Implementation and Integration. These comprise (1) complexity and (2) compatibility. Compared to the on premise approach, cloud systems can be implemented and running in 24 hours instead of six months [49]. Thus, implementation of cloud systems is not problematic for enterprises, whereas integrating cloud systems with the enterprise's existing IT infrastructure can negatively impact their adoption of cloud computing [49]. Further, the use of cloud system by IT staff is straightforward, while it is challenging for the non-IT staff. This can be attributed to the degree of complexity of cloud systems in terms of the ease of understanding, use, and implementation or integration of cloud services. Although cloud computing is considered to be easy to understand and use, it arouses integration complexity issues [50].

Complexity is claimed to be a barrier to the adoption of cloud computing [23, 50, $52,76]$. Integration complexity problems emerge from the less standardized interoperability between cloud systems and the existing IT infrastructure, which triggers integration costs [50]. A survey study reported that the lack of legacy systems allows enterprises to implement cloud computing easily [42]. The lack of compatibility of cloud solutions with existing IT infrastructure can be a barrier to the adoption of cloud computing [52]. Interoperability standards can be an enabler or a barrier to the adoption of cloud computing [70]; they are enablers when the enterprise has its data, processes, and systems standardize priori, but as technology evolves, it becomes challenging for the enterprise to catch change in technology. Thus, the enterprise faces a challenge to integrate cloud solutions with already-existing cloud-based or traditional systems. In order to ensure desirable implementation and integration of cloud systems, IT governance initiatives are a required.

IT Governance. This comprises (1) auditability and traceability and (2) risk management. Traceability and auditability are cited to have impact on cloud computing adoption, and the former complements the latter [49]. The loss of IT governance within the enterprise can slow down the adoption rates because the data and applications are under the control of the cloud provider [65, 83]. Enterprises are advised to conduct audit trail meetings with the cloud provider to ensure a risk-free implementation of cloud solutions that complies with regulations, standards, and enterprise policies [49]. This is enabled by the top management through IT governance structures and processes [50]. Contrarily, IT governance processes in highly regulated industries will decelerate the adoption of cloud computing [50]. Further, IT governance processes might hinder the adoption if the integration of cloud solutions with the existing infrastructure appears to be difficult [50]. 
Despite the massive advancements in securing the cloud, security solutions are not tested extensively yet [81]. This matter could be dissolved by IT governance initiatives to ensure that enterprise policies, security standards, and legal requirements are met $[49,50]$. Further, IT governance is attained by identifying responsibilities; for instance, the cloud provider may be responsible for the security at the IaaS level, whereas the customer's responsibility is at the SaaS and PaaS levels [83]. However, data security at the level of PaaS and IaaS service models can be a shared responsibility between the cloud provider and the adopting enterprise [66]. Additionally, cloud providers are required to provide traceable access controls to govern who can access what object under which conditions. This has to be validated by the top management for its conformity to internal and external constraints [49]. These controls are used to ensure data integrity and confidentiality $[49,65]$.

In regard to risk management during the planning for cloud computing implementation, a study suggests that the enterprise should consider evaluating the risk of storage damage, data loss, and network security [62]. For instance, the enterprise would maintain an on-site backup of the data moved to the cloud [69]. There is a lack of processes and methodologies that provides guidelines on how to use cloud services to address specific business needs and mitigate associated risks [71]. Eventually, securing enterprise's information from potential risks is more than processes, technical solutions, and people; it is an enterprise-wide security strategy to orchestrate these various elements [63].

Confirmation. This comprises usage continuance, about which a study proposed a model for implementation and confirmation stages of cloud computing adoption [79]. This study suggests the evaluation of cloud services based on the perceived attributes from using cloud services (i.e., relative advantage, complexity, compatibility, and trialability) to decide whether to continue using cloud services or not. Further, a survey study argued that perceived usefulness and satisfaction are necessary for IS continuance intention [43]. Apparently, satisfaction is not only related to perceived benefits from using cloud services, it is also dependent on the perception of service fairness from the cloud provider [43]. Service fairness happens when customers feel they are treated equally by the service provider as other customers are treated [43]. Thus, customers can judge how well-structured the cloud provider's system is, and, consequently, customers will likely continue to use the cloud provider's services [43].

\section{Discussion and Future Research Avenues}

This article sought to review 51 articles to capture the current state of IS research regarding cloud computing adoption in the context of enterprises. The review involved classifying the identified themes in the reviewed articles into cloud computing adoption factors and processes. The findings from the reviewed articles are discussed from three perspectives: theoretical (i.e., theories/frameworks/models utilized), methodological (i.e., research methods employed), and empirical (i.e., cloud computing adoption factors and processes). The contribution of this review is summarized in Fig. 2, where the identified cloud computing adoption factors and processes are depicted in addition to the relationships between them. In general, the review revealed 
that only three IS journal articles were found contributing to the area of cloud computing adoption issues. Likewise, few IS conference articles appeared to contribute to the understanding of cloud computing adoption. In contrast, articles from non-IS journal and conference outlets are dominant in investigating this phenomenon. Based on the findings, a cloud computing adoption research agenda is drawn accordingly to direct research avenues to towards (1) theoretical, (2) methodological, and (3) empirical studies.

\subsection{Theoretical Research Avenues}

Articles utilizing the grounded classification and general concepts are dominant, whereas few already-existing theories were utilized to study the adoption of cloud computing. Yet, there is a need for applying more theories (e.g., institutional theory $[90,91]$ ) that fit studying the adoption of IT innovation, to gain more insights regarding cloud computing adoption. Institutional theory captures the notion of irrationality in decision making, as enterprises may or may not adopt the cloud under internal (i.e., cultural resistance and internal readiness) or external pressure (i.e., competitors and business partners) and not because of efficiency and cost reduction. Moreover, institutional theory is helpful in understanding how enterprises respond to external and internal pressures and why [92, 93]. Consequently, this review brings interesting questions to IS researchers' empirical investigation briefcase: what factors (i.e., internal and external) affect the adoption of cloud computing, and how do enterprises form strategies to cope with these factors?

\subsection{Methodological Research Avenues}

The review indicates a lack of in-depth field and case studies regarding cloud computing adoption processes, as compared to those on cloud computing adoption factors, whereas the quantitative (i.e., surveys) studies and conceptual articles appeared to be dominant. Yet, there are theoretical studies that have not been tested. For instance, a study proposed theoretically a cloud computing adoption assessment model that considers criteria for selecting the cloud provider, but this model has not been tested empirically yet [67]. Thus, further qualitative research needs to be undertaken to explore further issues and test empirically the previous theoretical developments regarding this area.

Consequently, this triggers questions on the IS research round table as to why enterprises adopt cloud computing in spite of its potential risks? Or conversely, why enterprises do not adopt cloud computing in spite of its potential benefits? These questions need to be investigated thoroughly using multiple qualitative case studies in different contexts (i.e., countries and industries) to better understand cloud computing adoption factors and processes.

The majority of reviewed articles study cloud adoption factors and processes in a rather broad perspective. Therefore, there is a need for interpretive case studies to investigate each of cloud computing factors and processes found from this review (i.e., willingness, organizational culture, regulations, cloud providers trustworthiness, evaluation of cloud services, adoption decision, or implementation and integration processes) [94]. These in-depth studies are preferred owing to their implications for both practice and academia. 


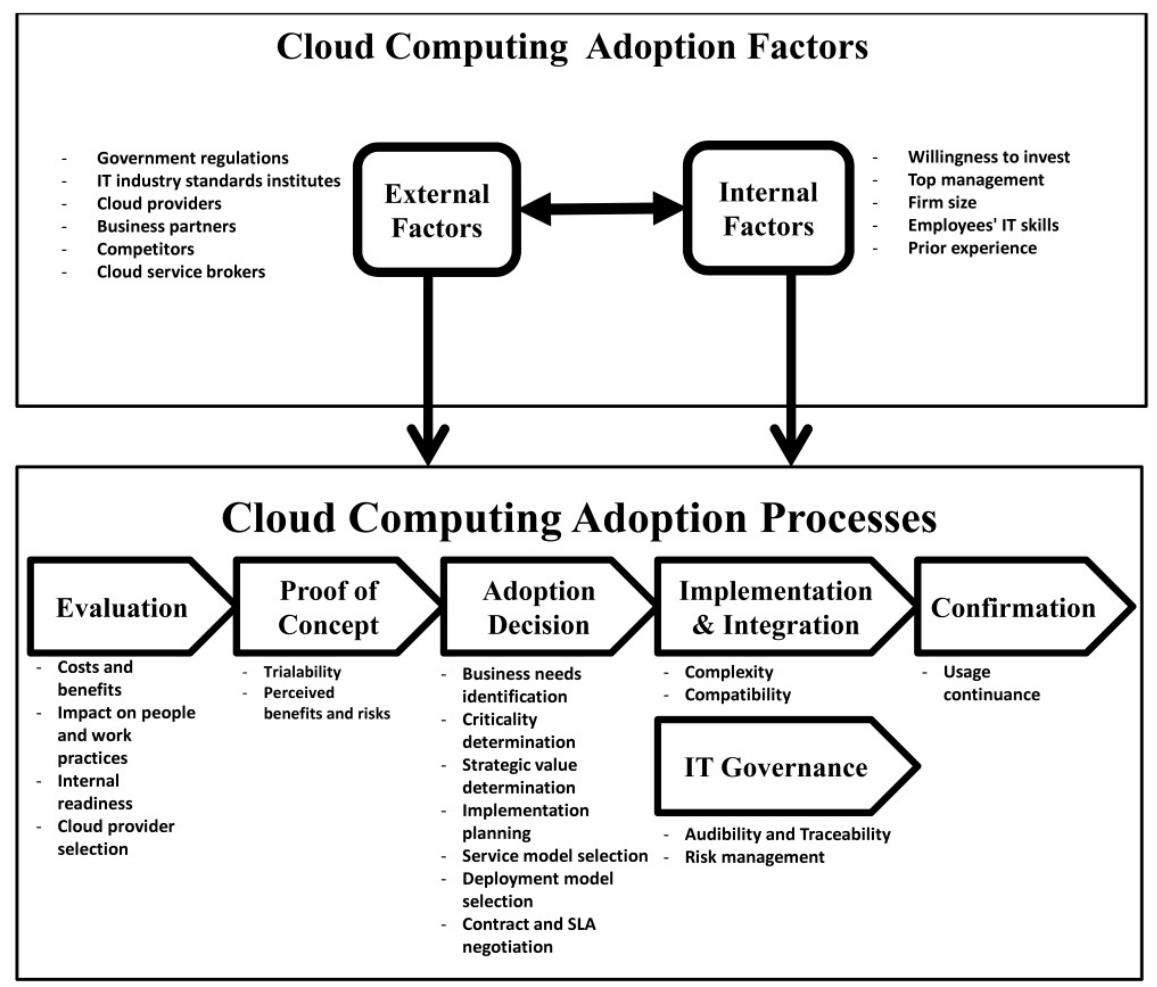

Fig. 2. Cloud computing adoption factors and processes

In spite of the appealing merits that cloud computing brings to enterprises (i.e., cost savings, flexibility, efficiency, agility and so forth), as an innovative IT shared services model [2], cloud computing puts enterprises in a decisive choice between on premise and on-demand approaches. In this regard, the Delphi method [95-97] would provide insights for IT managers about what are the most important issues, and their priorities, that should be considered when deciding to adopt cloud solutions. Further, this review advocates the need for conducting longitudinal studies to assess the impact of cloud computing implementation on both the technical and managerial capabilities of the enterprise (i.e., integration with existing IT infrastructure, planning, risk management, and IT governance) as well as the impact of the confirmation process on the organizational innovation.

\subsection{Empirical Research Avenues}

The review shows that cloud computing adoption processes received less attention from IS researchers in terms of exploring the challenges faced in each process and how enterprises cope with these challenges for risk-free adoption of cloud services. Legal issues are taking most of the attention in terms of the adequacy and consistency of government regulations for ensuring security and data privacy needed for using cloud computing services. In the developed world, these regulations, either national or 
international, are in place, but they are inconsistent with each other, whereas, in the developing, world there is a lack of regulatory frameworks to assure safe adoption of cloud computing services. This implies the need for exploring cloud computing adoption in developing countries, as they may lack legal and IT infrastructures [78, 98], and the need for transferring lessons from the developed world to the developing one and vice versa.

According to the review, although cloud computing adoption factors have been investigated slightly more than the processes have, plenty of issues remain unclear. For instance, there is a conflict regarding the relationship between the firm's size and the likelihood to adopt cloud computing, and further in-depth studies are needed to address this conflict. Further, cloud computing is recognized as a cost-reduction solution; however, this cost reduction may not be significant, particularly in the context of SMEs, as reported by a survey study conducted in India [86]. Likewise, when the enterprise maintains an on premise backup, this adds to the cost as well [69]. If cloud computing helps enterprises reduce IT-related costs, then how significant will be this cost reduction be? Thus, further studies with focus on evaluating costs and benefits of cloud computing solutions would be favorable.

Moreover, there is a need for further exploring the impact of IT governance processes throughout the implementation process. There are still many legal (i.e., contract and SLA), ethical, and inter-organizational or institutional issues that need to be investigated regarding improvements of laws and standards. In addition, there is a need to explore the role of cloud service brokers in enabling cloud computing adoption and whether they have sufficient service management tools for provisioning cloud services or not. Moreover, trust issues are not addressed extensively in the reviewed articles, although it is claimed to be important for the successful adoption of cloud computing [3].

Further, it would be useful to investigate internal readiness and selection of cloud provider issues in the context of SMEs and/or large enterprises. Future studies should explore the compatibility between cloud solutions with enterprises' legacy systems and business needs, as well as the impact of trying or using cloud solutions on organizational culture, staff skills, and work practices. Another issue to consider is whether cloud computing releases enterprises from managing the IT infrastructure so they can focus on their core business activities, and if so, which data and applications should be moved to the cloud and which should remain in-house? Further studies would be appropriate for providing recommendations for practice regarding internal preparation, service model selection, and contract negotiation issues.

\section{Conclusion}

This study sought to conduct a systematic review of the extant literature on cloud computing adoption by enterprises. This involved identifying the current contributions of IS research regarding the phenomenon and determining the underinvestigated issues and the contributions of IS research regarding the phenomenon. The classification of reviewed articles, findings, and implications for future IS research avenues are according to theories, research methods, and cloud computing adoption factors and processes that were identified by using GT approach. Yet, plentiful 
legal, ethical, technical, and managerial issues are waiting for IS researchers to explore. Thus, the paper suggested a future IS research agenda based on the discussed findings.

This article is not free of limitations; it sought to review only academic articles from seven literature databases, leaving out white papers, magazine articles, other scholarly literature databases, and articles from a forward and backward search, the inclusion of which would help capture more issues about cloud computing adoption by enterprises. The search criterion was limited to article title only; however, including abstracts as a criterion would have revealed more insightful articles. The search phrases were limited; as some articles discuss cloud computing adoption using different words (i.e., utility computing or application service provision) that may not have been included in the search results of this review.

Acknowledgements. This paper is made possible through the help and support from Dr. Eli Hustad, Prof. Dag H. Olsen, Prof. Peter Axel Nielsen, and my colleagues Moutaz Haddara, Fathul Wahid, and Christoph Merschbrock. I owe them thanks for offering valuable advices on the logic and organization of the paper at its early stages. Finally, I sincerely thank the anonymous reviewers for their constructive advices that made the paper appear in a well-presented final shape.

\section{References}

1. Elragal, A., Haddara, M.: The Future of ERP Systems: Look Backward Before Moving Forward. Procedia Technol. 5, 21-30 (2012)

2. Su, N., Akkiraju, R., Nayak, N., Goodwin, R.: Shared Services Transformation: Conceptualization and Valuation from the Perspective of Real Options. Decis. Sci. 40, 381-402 (2009)

3. Garrison, G., Kim, S., Wakefield, R.L.: Success Factors for Deploying Cloud Computing. Commun. ACM. 55, 62-68 (2012)

4. Parakala, K., Udhas, P.: The Cloud Changing the Business Ecosystem (2011), http: / / www . kpmg.com/IN/en/IssuesAndInsights/ThoughtLeadershi p/The_Cloud_Changing_the_Business_Ecosystem.pdf

5. Herhalt, J., Cochrane, K.: Exploring the Cloud: A Global Study of Governments' Adoption of Cloud (2012),

http: / / www . kpmg. com/ES/es / ActualidadyNovedades / ArticulosyPub licaciones / Documents / Exploring-the-Cloud.pdf

6. Venters, W., Whitley, E.A.: A Critical Review of Cloud Computing: Researching Desires and Realities. J. Inf. Technol. 27, 179-197 (2012)

7. RightScale: RightScale State of the Cloud Report (2013),

https://www.rightscale.com/pdf/ rightscale-state-of-the-cloud-report-2013.pdf

8. Armbrust, M., Fox, A., Griffith, R., Joseph, A.D., Katz, R., Konwinski, A., Lee, G., Patterson, D., Rabkin, A., Stoica, I., Zaharia, M.: Above the Clouds A Berkeley View of Cloud Computing (2009)

9. Sultan, N.A.: Reaching for the "Cloud": How SMEs Can Manage. Int. J. Inf. Manage. 31, 272-278 (2011)

10. Yang, H., Tate, M.: A Descriptive Literature Review and Classification of Cloud Computing Research. Commun. Assoc. Inf. Syst. 31 (2012)

11. Cummings, M.P., Huskamp, J.C.: Grid Computing. Educ. Rev. 40, 116-117 (2005) 
12. Weinhardt, C., Anandasivam, A., Blau, B., Borissov, N., Meinl, T., Michalk, W., Stößer, J.: Cloud Computing - A Classification, Business Models, and Research Directions. Bus. Inf. Syst. Eng. 1, 391-399 (2009)

13. EMA: Hybrid IT Service Management: A Requirement for Virtualization and Cloud Computing (2012),

http: / / www. thinkhdi.com/ /media/HDICorp/Files/ White-Papers/whtppr-FrontRange-Hybrid-ITSM.pdf

14. Heiser, J., Nicolett, M.: Assessing the Security Risks of Cloud Computing (2008)

15. Hashemi, S.M., Bardsiri, A.K.: Cloud Computing Vs. Grid Computing. ARPN J. Syst. Softw. 2, 188-194 (2012)

16. Susarla, A., Barua, A., Whinston, A.: Understanding the Service Component of Application Service Provision: An Empirical Analysis of Satisfaction with ASP Services. MIS Q. 27, 91-123 (2003)

17. Kern, T., Willcocks, L.P., Lacity, M.C.: Application Service Provision Risk Assessment and Mitigation. MIS Q. Exec. 1, 113-126 (2002)

18. Robison, S.: Everything-as-a-Service: A Blue Sky View of the Cloud (2009), http://www.hp.com/hpinfo/initiatives/eaas/SR_EaaS_viewpoint.pdf

19. Xin, L., Song, C.: Cloud-Based Innovation of Internet Long Tail. In: The 6th International Conference on Product Innovation Management (ICPIM 2011), pp. 603-607 (2011)

20. Kunesch, U., Reti, M., Pauly, M.: Cloud Computing I. Alternative sourcing Strategy for Business ICT (2010), http://www.t-systems.com/news-media/whitepapers/760948_2/blobBinary/White-Paper_Cloud-Computing-I.pdf

21. Mell, P., Grance, T.: The NIST Definition of Cloud Computing: Recommendations of the National Institute of Standards and Technology (2011),

http://csrc.nist.gov/publications/nistpubs/800-145/

SP800-145.pdf

22. Hogan, M., Liu, F., Sokol, A., Tong, J.: NIST Cloud Computing Standards Roadmap (2011), http://www.nist.gov/customcf/get_pdf.cfm?pub_id=909024

23. Espadanal, M., Oliveira, T.: Cloud Computing Adoption by Firms. In: Proceedings of the Mediterranean Conference on Information Systems, MCIS 2012 (2012)

24. Yang, H., Jing, S., Wang, H.: The Research on ASP-based SMEs Collaborative Platform. In: Proceedings of the 8th International Conference on Computer Supported Cooperative Work in Design, vol. 2, pp. 6-9 (2004)

25. Malathi, M.: Cloud computing Concepts. In: 2011 3rd International Conference on Electronics Computer Technology (ICECT), pp. 236-239 (2011)

26. Salleh, S.M., Teoh, S.Y., Chan, C.: Cloud Enterprise Systems A Review of Literature and its Adoption. In: Proceedings of the Pacific Asia Conference on Information Systems, PACIS 2012 (2012)

27. Marston, S., Li, Z., Bandyopadhyay, S., Zhang, J., Ghalsasi, A.: Cloud computing - The Business Perspective. Decis. Support Syst. 51, 176-189 (2011)

28. Timmermans, J., Stahl, B.C., Ikonen, V., Bozdag, E.: The Ethics of Cloud Computing: A Conceptual Review. In: Proceedings of the Second International Conference on Cloud Computing Technology and Science, pp. 614-620. IEEE (2010)

29. Webster, J., Watson, R.: Analysing the Past to Prepare for the Future: Writing a Literature Review. MIS Q. 26, xiii-xxiii (2002)

30. Vom Brocke, J., Simons, A., Niehaves, B., Riemer, K., Plattfaut, R., Cleven, A.: Reconstructing the Giant: On the Importance of Rigour in Documenting the Literature Search Process. In: The 17th European Conference on Information Systems (ECIS 2009), Verona, Italy, pp. 2206-2217 (2009) 
31. Levy, Y., Ellis, T.J.: A Systems Approach to Conduct an Effective Literature Review in Support of Information Systems Research. Informing Sci. J. 9, 181-212 (2006)

32. Dubey, A., Wagle, D.: Delivering Software as a Service (2007)

33. Okoli, C., Schabram, K.: A Guide to Conducting a Systematic Literature Review of Information Systems Research. Sprouts Work. Pap. Inf. Syst. 10 (2010)

34. Orlikowski, W.J., Baroudi, J.J.: Studying Information Technology in Organizations: Research Approaches and Assumptions. Inf. Syst. Res. 2, 1-28 (1991)

35. Galliers, R.D., Land, F.F.: Choosing Systems Appropriate Research Information Methodologies. Commun. ACM. 30, 900-902 (1987)

36. Gonzalez, R., Dahanayake, A.: A Concept Map of Information Systems Research Approaches. In: Proceedings of the IRMA International Conference, pp. 845-849 (2007)

37. Glaser, B.G., Strauss, A.L.: The Discovery of Grounded Theory: Strategies for Qualitative Research. Aldine Publishing Company, Chicago (1967)

38. Wolfswinkel, J.F., Furtmueller, E., Wilderom, C.P.M.: Using Grounded Theory as a Method for Rigorously Reviewing Literature. Eur. J. Inf. Syst. 22, 45-55 (2011)

39. Rich, P.: Inside the Black BoxRevealing the Process in Applying a Grounded Theory Analysis. Qual. Rep. 17, 1-23 (2012)

40. Bharadwaj, S.S., Lal, P.: Exploring the Impact of Cloud Computing Adoption on Organizational Flexibility: A Client Perspective. In: Proceedings of the International Conference on Cloud Computing Technologies, Applications and Management (ICCCTAM 2012), pp. 121-131. IEEE (2012)

41. Opitz, N., Langkau, T.F., Schmidt, N.H., Kolbe, L.M.: Technology Acceptance of Cloud Computing: Empirical Evidence from German IT Departments. In: Proc. 45th Hawaii Int. Conf. Syst. Sci. (HICSS 2012), pp. 1593-1602 (2012)

42. Tjikongo, R., Uys, W.: The Viability of Cloud Computing Adoption in SMME's in Namibia. In: Proceedings of IST-Africa Conference, pp. 1-11 (2013)

43. Lawkobkit, M., Speece, M.: Integrating Focal Determinants of Service Fairness into PostAcceptance Model of IS Continuance in Cloud Computing. In: Proc. 11th Int. Conf. Comput. Inf. Sci. (2012 IEEE/ACIS), pp. 49-55 (2012)

44. Shin, J., Jo, M., Lee, J., Lee, D.: Strategic Management of Cloud Computing Focusing on Consumer Adoption Behavior. IEEE Trans. Eng. Manag., 1-9 (2013)

45. Lian, J.-W., Yen, D.C., Wang, Y.-T.: An Exploratory Study to Understand the Critical Factors Affecting the Decision to Adopt Cloud Computing in Taiwan Hospital. Int. J. Inf. Manage. 34, 28-36 (2014)

46. Bogataj, K., Pucihar, A.: Business Model Factors Influencing Cloud Computing Adoption Differences in Opinion. In: Proceedings of the BLED 2013, pp. 1-14 (2013)

47. Kusnandar, T., Surendro, K.: Adoption Model of Hospital Information System Based on Cloud Computing Case Study on Hospitals in Bandung City. In: Proceedings of the International Conference on ICT for Smart Society, pp. 1-6 (2014)

48. Saedi, A., Iahad, N.A.: An Integrated Theoretical Framework for Cloud Computing Adoption by Small and Medium-sized Enterprises. In: Proceedings of the Pacific Asia Conference on Information Systems (PACIS 2013), pp. 1-12 (2013)

49. Morgan, L., Conboy, K.: Factors Affecting the Adoption of Cloud Computin An Exploratory Study. In: Proceedings of the 21st European Conference on Information Systems, pp. 1-13 (2013)

50. Borgman, H.P., Bahli, B., Heier, H., Schewski, F.: Cloudrise: Exploring Cloud Computing Adoption and Governance with the TOE Framework. In: Proceedings of the 46th Hawaii International Conference on System Sciences, pp. 4425-4435. IEEE (2013) 
51. Alshamaila, Y., Papagiannidis, S.: Cloud computing Adoption by SMEs in the North East of England: A Multi-perspective Framework. J. Enterp. Inf. Manag. 26, 250-275 (2013)

52. Low, C., Chen, Y., Wu, M.: Understanding the Determinants of Cloud Computing Adoption. Ind. Manag. Data Syst. 111, 1006-1023 (2011)

53. Alshamaila, Y., Papagiannidis, S., Stamati, T.: Cloud Computing Adoption in Greece. In: Proceedings of UK Academy for Information Systems Conference, pp. 1-17 (2013)

54. Nkhoma, M.Z., Dang, D.P.T., De Souza-daw, A.: Contributing Factors of Cloud Computing Adoption A Technology- Organisation-Environment Framework Approach. In: Proceedings of the European Conference on Information Management \& Evaluation, pp. 180-189 (2013)

55. Saedi, A., Iahad, N.A.: Developing an Instrument for Cloud Computing Adoption by Small and Medium-sized Enterprises. In: Proceedings of the International Conference on Research and Innovation in Information Systems (ICRIIS 2013), pp. 481-486. IEEE (2013)

56. Chang, B.-Y., Hai, P.H., Seo, D.-W., Lee, J.-H., Yoon, S.H.: The Determinant of Adoption in Cloud Computing in Vietnam. In: Proceedings of the International Conference on Computing, Management and Telecommunications (ComManTel 2013), pp. 407-409. IEEE (2013)

57. Cegielski, C.G., Jones-Farmer, L.A., Wu, Y., Hazen, B.T.: Adoption of Cloud Computing Technologies in Supply Chains: An Organizational Information Processing Theory Approach. Int. J. Logist. Manag. 23, 184-211 (2012)

58. Yang, C., Hwang, B., Yuan, B.J.C.: Key Consideration Factors of Adopting Cloud Computing for Science. In: Proceedings of the 4th International Conference on Cloud Computing Technology and Science, pp. 597-600 (2012)

59. Stankov, I., Miroshnychenko, Y., Kurbel, K.: Cloud Computing Adoption in German Internet Start-up Companies. In: Proceedings of BLED Conference, BLED 2012 (2012)

60. Rath, A., Mohapatra, S., Kumar, S., Thakurta, R.: Decision Points for Adoption Cloud Computing in Small, Medium Enterprises ( SMEs ). In: Proceedings of the 7th International Conference for Internet Technology and Secured Transactions (ICITST 2012), pp. 688-691 (2012)

61. Abokhodair, N., Taylor, H., Hasegawa, J., Mowery, S.J.: Heading for the Clouds? Implications for Cloud Computing Adopters. In: Proceedings of the 18th Americas Conference on Information Systems, AMCIS 2012 (2012)

62. Tsai, C., Lin, U.-C., Chang, A.Y., Chen, C.-J.: Information Security Issue of Enterprises Adopting the Application of Cloud Computing. In: Proceedings of the 6th International Conference on Networked Computing and Advanced Information Management (NCM 2010), pp. 645-649 (2010)

63. Opala, O.J., Rahman, S.(Shawon)M.: An Exploratory Analysis of the Influence of Information Security on the Adoption of Cloud Computing. In: Proceedings of the 2013 8th International Conference on System of Systems Engineering, pp. 165-170 (2013)

64. Subramanian, B.: The Disruptive Influence of Cloud Computing and its Implications for Adoption in the Pharmaceutical and Life Sciences Industry. J. Med. Mark. Device, Diagnostic Pharm. Mark. 12, 192-203 (2012)

65. Onwudebelu, U., Chukuka, B.: Will Adoption of Cloud Computing Put the Enterprise at Risk? In: Proceedings of the 4th International Conference on Adaptive Science \& Technology (ICAST 2012), pp. 82-85. IEEE (2012) 
66. Bamiah, M., Brohi, S., Chuprat, S., Ab Manan, J.: A Study on Significance of Adopting Cloud Computing Paradigm in Healthcare Sector. In: Proceedings of the International Conference on Cloud Computing Technologies, Applications and Management (ICCCTAM 2012), pp. 65-68. IEEE (2012)

67. Nasir, U., Niazi, M.: Cloud Computing Adoption Assessment Model (CAAM ). In: Proceedings of the 12th International Conference on Product Focused Software Development and Process Improvement, pp. 34-37 (2011)

68. Jensen, M., Schwenk, J., Bohli, J.-M., Gruschka, N., Iacono, L.L.: Security Prospects Through Cloud Computing by Adopting Multiple Clouds. In: Proceedings of the 4th International Conference on Cloud Computing (CLOUD), pp. 565-572. IEEE (2011)

69. Kim, W., Kim, S.D., Lee, E., Lee, S.: Adoption Issues for Cloud Computing. In: Proceedings of the 11th International Conference on Information Integration and Web-based Applications \& Services - iiWAS 2009, p. 2. ACM Press, New York (2009)

70. Avram, M.G.: Advantages and Challenges of Adopting Cloud Computing from an Enterprise Perspective. Procedia Technol. 12, 529-534 (2014)

71. Bellamy, M.: Adoption of Cloud Computing Services by Public Sector Organisations. In: Proceedings of the IEEE 9th World Congress on Services, pp. 201-208. IEEE (2013)

72. Kushida, K.E., Murray, J., Zysman, J.: Diffusing the Cloud: Cloud Computing and Implications for Public Policy. J. Ind. Compet. Trade. 11, 209-237 (2011)

73. Rawal, A.: Adoption of Cloud Computing in India. J. Technol. Manag. Grow. Econ. 2, 65-78 (2011)

74. Coursaris, C.K., van Osch, W., Sung, J.: A "Cloud Lifestyle": The Diffusion of Cloud Computing Applications and the Effect of Demographic and Lifestyle Clusters. In: Proceedings of the 46th Hawaii International Conference on System Sciences (HICSS 2013), pp. 2803-2812. IEEE (2013)

75. Malladi, S., Krishnan, M.S.: Cloud Computing Adoption and its Implications for CIO Strategic Focus - An Empirical Analysis. In: Proceedings of 33rd International Conference on Information Systems (ICIS 2012), pp. 1-19 (2012)

76. Lin, A., Chen, N.-C.: Cloud Computing as an Innovation: Percepetion, Attitude, and Adoption. Int. J. Inf. Manage. 32, 533-540 (2012)

77. Nuseibeh, H.: Adoption of Cloud Computing in Organizations. In: Proceedings of the 17th Americas Conference on Information Systems, AMCIS 2011 (2011)

78. Bhat, J.M.: Adoption of Cloud Computing by SMEs in India: A study of the Institutional Factors. In: Proceedings of the 19th Americas Conference on Information Systems, pp. 1-8 (2013)

79. Dargha, R.: Cloud Computing From Hype to Reality. Fast Tracking Cloud Adoption. In: Proceedings of the Intermational Conference on Advances in Computing, Communications, and Informatics, pp. 440-445 (2012)

80. Hoe, S.C., Kantarcioglu, M., Bensoussan, A.: Studying Dynamic Equilibrium of Cloud Computing Adoption with Application of Mean Field Games. In: Proceedings of the 50th Annual Allerton Conference on Communication, Control, and Computing (Allerton), pp. 220-224. IEEE (2012)

81. Misra, S.C., Mondal, A.: Identification of a Company's Suitability for the Adoption of Cloud Computing and Modelling its Corresponding Return on Investment. Math. Comput. Model. 53, 504-521 (2011)

82. Kantarcioglu, M., Bensoussan, A., Hoe, S.C.: Impact of Security Risks on Cloud Computing Adoption. In: Proceedings of the 49th Annual Allerton Conference on Communication, Control, and Computing (Allerton), pp. 670-674. IEEE (2011) 
83. Mcgeogh, B.T., Donnellan, B.: Factors That Affect the Adoption of Cloud Computing for an Enterprise A Case Study of Cloud Adoption Within Intel Corporation. In: Proceedings of the 21st European Conference on Information Systems, pp. 1-13 (2013)

84. Zhao, F., Gaw, S.D., Bender, N., Levy, D.T.: Exploring Cloud Computing Adoptions in Public Sectors: A Case Study. GSTF J. Comput. 3 (2013)

85. Gupta, P., Seetharaman, A., Raj, J.R.: The Usage and Adoption of Cloud Computing by Small and Medium Businesses. Int. J. Inf. Manage. 33, 861-874 (2013)

86. Iyer, E.K., Krishnan, A., Sareen, G., Panda, T.: Analysis of Dissatisfiers That Inhibit Cloud Computing Adoption Across Multiple Customer Segments. In: Proceedings of the European Conference on Information Management \& Evaluation, pp. 145-152 (2013)

87. Luoma, E., Nyberg, T.: Four Scenarios for Adoption of Cloud Computing in China. In: Proceedings of the European Conference on Information Systems, ECIS 2011 (2011)

88. Khanagha, S., Volberda, H., Sidhu, J., Oshri, I.: Management Innovation and Adoption of Emerging Technologies: The Case of Cloud Computing. Eur. Manag. Rev. 10, 51-67 (2013)

89. Kihara, T., Gichoya, D.: Adoption and Use of Cloud Computing in Small and Medium Enterprises in Kenya. In: Proceedings of IST-Africa Conference, pp. 1-12 (2013)

90. Mignerat, M., Rivard, S.: Positioning the Institutional Perspective in Information Systems Research. J. Inf. Technol. 24, 369-391 (2009)

91. Weerakkody, V., Dwivedi, Y.K., Irani, Z.: The Diffusion and Use of Institutional Theory: A Cross-disciplinary Longitudinal Literature Survey. J. Inf. Technol. 24, 354-368 (2009)

92. Oliver, C.: Strategic Responses to Institutional Processes. Acad. Manag. Rev. 16, 145-179 (1991)

93. Deephouse, D.L., Suchman, M.: Legitimacy in Organizational Institutionalism. In: Greenwood, R., Oliver, C., Suddaby, R., Sahlin, K. (eds.) The SAGE Handbook of Organizational Institutionalism, pp. 49-77. SAGE Publications Ltd., Thousand Oaks (2008)

94. Walsham, G.: Interpretive Case Studies in IS Research Nature and Method. Eur. J. Inf. Syst. 4, 74-81 (1995)

95. Dalkey, N.C.: The Delphi Method: An Experimental Study of Group Opinion. In: Dalkey, N.C. (ed.) Studies in the Quality of Life: Delphi and Decision-Making, p. 161. Lexington Books (1972)

96. Okoli, C., Pawlowski, S.D.: The Delphi Method as a Research Tool An Example, Design Considerations and Applications. Inf. Manag. 42, 15-29 (2004)

97. Hsu, C., Sandford, B.A.: The Delphi Technique: Making Sense of Consensus. Pract. Assessment, Res. Eval. 12, 1-8 (2007)

98. Greengard, S.: Cloud Computing and Developing Nations. Commun. ACM 53, 18 (2010) 